\title{
Does uncertainty effect the case of exhaustive interpretation?
}

Charlotte Wollermann ${ }^{1}$ and Bernhard Schröder ${ }^{2}$

${ }^{1}$ Department of Speech and Communication, University of Bonn, Germany

${ }^{2}$ German Linguistics, University of Essen/Duisburg, Germany

https://doi.org/10.36505/ExLing-2008/02/0059/000118

\begin{abstract}
We present an experimental study investigating the role of intonation as prosodic indicator of uncertainty as well as question-answering congruity for the exhaustive interpretation of answers. We vary intonation for expressing intended (un)certainty and also the type of question. Interpretation is tested by using pictures illustrating (non-)exhaustivity in order to avoid that the subjects' linguistic awareness is focussed on the tested question.
\end{abstract}

Key words: exhaustive interpretation, focus detection, uncertainty, intonation, question-answering congruity

\section{Introduction}

If the hearer concludes from (1b) that John and Mary are the only persons out of a number of people in question who passed the examination, the predicate in question in (1a) is interpreted exhaustively (cf. van Rooij, Schulz 2006: 205). In the case of non-exhaustive interpretation there are (or may be) also other persons who passed the examination.

1a Who passed the examination?

1b John and Mary.

According to semantic-pragmatic theories (e.g. Groenendijk, Stokhof 1984; Rooth 1992) in a context of a question, accent is highly correlated with focus. This should have a clear impact on exhaustive interpretation, especially in the context of a suitable question. An exhaustive interpretation is dependent on the knowledge about the situation in question, as ascribed to the speaker by the hearer. If the speaker is believed to have only uncertain knowledge of a situation, the hearer will less attract an exhaustive interpretation.

Several studies found that rising intonation is one of the acoustic cues which contribute to the perception of uncertainty (e.g. Smith, Clark 1993; Swerts et al. 2003). The analysis of Ward and Hirschberg (cf. 1985: 747) showed that fall-rise intonation contributes to a context-independent meaning of utterance interpretation conveying speaker's uncertainty ${ }^{1}$.

ExLing 2008: Proceedings of 2nd Tutorial and Research Workshop on Experimental Linguistics, 25-27 August 2008, Athens, Greece 
However, there is barely empirical evidence of the role of uncertainty for exhaustive interpretation.

\section{Experimental study}

\section{Goal}

The goal of our experiment is to investigate whether intonation as prosodic indicator of uncertainty and the type of question effect exhaustive interpretation of answers.

\section{Method}

Our audio stimuli consist of question-answer pairs, which are embedded into dialogs. Altogether there are six dialogs. The scenario is a fictional student dress-up party, where different student groups carry out different actions.

The focus of the answer is each time a noun phrase. It is either marked by fall-rise intonation (expressing uncertainty), or falling intonation (expressing certainty). The preceding question is either syntactically and lexically parallel to the answer or constitutes a more general question.

Our hypothesis is that falling intonation combined with parallel question favors exhaustive interpretation, whereas fall-rise intonation combined with general question advantages non-exhaustive interpretation. Independent variables are thus intonation and the type of question, the dependent variable is exhaustivity.

For testing the interpretation, subjects have to choose between different pictures ${ }^{2}$. One picture illustrating exhaustive interpretation, another showing non-exhaustive reading and a third picture showing an irrelevant scene functioning as distractor. Each group is characterized by different accessories, which is illustrated by the pictures. Also as distractor, we ask questions about the subjects' personal opinion of an aspect of the dialog and use two filler-dialogs ${ }^{3}$.

Subjects are 71 students (24 males, 47 females) of the Institute of Communication Sciences, all native speakers of German. They are tested in four group experiments: Subsets of the dialogs are presented each time with a different kind of random order of the stimuli and also of the pictures. We use Fisher's exact test to examine the significance of the association between two categorical variables.

\section{Results}

Results are presented in Table 1. As the table shows most interpretations are exclusively exhaustive, but there are also some non-exhaustive interpretations. The number varies according to the combinations and the dialogs. Significant differences are as follows: For Dialog 2 the comparison 
of general question combined with certainty $(\mathrm{C})$ and general question plus uncertainty (D) yields a significant difference with $\mathrm{p}=0.01$. Here, intonation seems to clearly influence the judgments.

For Dialog 4 general question plus certainty (C) is judged as more nonexhaustive than certainty combined with congruent question $(A)(p=0.02)$. Thus, question-answering congruity favors exhaustivity in this case. In accordance, general question joined with uncertainty (D) obtains more nonexhaustive readings than congruent question plus certainty (A) with $p=0.049$.

Table 1. Amount of answers in both categories of exhaustivity. Abbreviations: Exh+: Exclusively exhaustive interpretations, Exh-: Readings that comprehend non-exhaustive interpretation, $C Q$ : Congruent question, $G Q$ : General question, $C$ : Certainty, $U$ : Uncertainty. Significant different judgments are marked in bold face.

\begin{tabular}{|l|l|l|l|l|l|l|l|l|l|}
\hline Stimulus & Description & D1 & \multicolumn{1}{|l|}{ D2 } & D3 & \multicolumn{2}{l|}{ D4 } \\
\hline & Exh. & + & - & + & - & + & - & + & - \\
\hline A & CQ + C & 14 & 4 & 17 & 2 & 9 & 6 & 15 & 0 \\
\hline B & CQ +U & 12 & 3 & 16 & 3 & 17 & 2 & 17 & 1 \\
\hline C & GQ+C & 12 & 6 & 18 & 0 & 12 & 7 & 9 & 5 \\
\hline D & GQ+U & 14 & 9 & 10 & 5 & 16 & 2 & 13 & 5 \\
\hline
\end{tabular}

\begin{tabular}{|l|l|l|l|l|l|}
\hline Stimulus & Description & D5 & \multicolumn{2}{l|}{ D6 } \\
\hline & Exh. & + & - & + & - \\
\hline A & CQ+C & 16 & 3 & 15 & 4 \\
\hline B & CQ+U & 8 & 7 & 16 & 2 \\
\hline C & GQ + C & 13 & 6 & 16 & 2 \\
\hline D & GQ+U & 16 & 2 & 10 & 5 \\
\hline
\end{tabular}

For Dialog 5 we have more non-exhaustive interpretations for congruent question joined with uncertainty (B) than with certainty (A). Differences are marginally significant $(p=0.068)$. We assume that intonation influences exhaustivity here. But surprisingly, general question with uncertainty (D) is ranked as more exhaustive than (B) with $p=0.047$. Here, the tendency does not mirror what was expected theoretically. This could possibly be explained by the fact that in this single case the fall-rise intonation is realised more conspicuous than the falling intonation and thus favors exhaustivity.

We assume that for Dialog 2, 4 and 5 as our three "successful" dialogs, intonation and linguistic context (i.e. choice of the verbs) is realised more adequate than for the remaining three "unsuccessful" dialogs. 


\section{Discussion}

The experiment brought to light that exhaustive reading is considered as standard interpretation in our scenario. However, in three of six dialogs, results suggest that exhaustivity can be influenced by question-answering congruity and also by intonation. But our data reveal that intonation exclusively does not have such a strong effect on exhaustivity as semanticpragmatic theories would suggest.

Future work will concentrate on the "successful" dialogs. We will investigate if additional prosodic cues indicating uncertainty like pauses and a hesitant way of speaking combined with fall-rise intonation and/or contextual factors facilitate non-exhaustive interpretation.

\section{Notes}

${ }^{1}$ Fall-rise intonation is defined as follows: Firstly, the pitch peak is reached late in the accented syllable and a relatively abrupt drop in pitch must appear in the two following syllables. Secondly, a sentence-final rise in pitch is at hand (cf. Ward, Hirschberg 1985: 748).

${ }^{2}$ For each dialog, the pictures and questions are presented on slides and presented via a beamer, subjects only have to mark their choice on a handed-out questionnaire. In this way, we want to assure that subjects do not page back and change their judgments.

${ }^{3}$ Subjects have the possibility to choose multiple answers for both tasks.

\section{Acknowledgements}

We would like to thank Petra Wagner, Ulrich Schade, Eva Lasarcyk, and Bernhard Fisseni for helpful discussions and comments. Many thanks go to our speakers Julia Abresch and Stefan Keller.

\section{References}

Groenendijk, J. and Stokhof, M. 1984. Studies on the Semantics of Questions and the Pragmatics of Answers. Dissertation, University of Amsterdam.

Rooth, M. 1992. A theory of focus interpretation. In Natural Language Semantics, 75-116.

Smith, V. and Clark, H. 1993. On the course of answering questions. In Journal of Memory and Language, 32, 25-38.

Swerts, M., Krahmer, E., Barkhuysen, P. and van de Laar, L. 2003. Audiovisual cues to uncertainty. In Proceedings of ISCA Workshop on Error Handling in Spoken Dialog Systems, Chateau-d'Oex, Switzerland.

van Rooij, R. and Schulz, K. 2006. Pragmatic Meaning and Non-monotonic Reasoning: The Case of Exhaustive Interpretation. In Linguistics and Philosophy, 29, 205-250.

Ward, G. and Hirschberg, J. 1985. Implicating Uncertainty: The Pragmatics of FallRise Intonation. In Language, 61(4), 747-776. 